\title{
Anti-periodic solutions for BAM-type Cohen-Grossberg neural networks with time delays
}

\author{
Ping Cui ${ }^{\mathrm{a}, *}$, Zheng-Biao $\mathrm{Li}^{\mathrm{b}}$ \\ aInstitute of Applied Mathematics, School of Teacher Education, Qujing Normal University, Qujing Yunnan, 655011, China. \\ ${ }^{b}$ School of Mathematics and Statistics, Qujing Normal University, Qujing Yunnan, 655011, China.
}

Communicated by $\mathrm{X}$. J. Yang

\begin{abstract}
In this paper, a class of BAM-type Cohen-Grossberg neural networks with time delays are considered. Some sufficient conditions for the existence and exponential stability of anti-periodic solutions are established. (C)2017 All rights reserved.
\end{abstract}

Keywords: BAM Cohen-Grossberg neural networks, time delay, anti-periodic solution, exponential stability. 2010 MSC: 34C25, 34D23, 37C75.

\section{Introduction}

In recent years, Cohen and Grossberg neural networks [5] have been extensively studied and applied in many different fields such as associative memory, signal processing and some optimization problems. The bidirectional associative memory (BAM) model know as an extension of the unidirectional autoassociator of Hopfield [9], was first introduced by Kosto [11]. This neural network has been widely studied due to its promising potential for applications in pattern recognition and automatic control.

Continuous bidirectional associative memory (BAM) is made up of two (or more) neural fields $F_{x}$ and $F_{y}$, connected in the forward direction, from $F_{x}$ to $F_{y}$, by an arbitrary $n$-by-p synaptic matrix $M$ and connected in the backward direction, from $F_{y}$ to $F_{x}$, by the p-by-n matrix N. In [11, 12], Kosto has proposed bidirectional associative memory neural networks with and without axonal signal transmission delays. In [5], Cohen and Grossberg have studied the following BAM model that possesses CohenGrossberg dynamics, and their extension can be described as follows:

$$
\left\{\begin{array}{l}
\frac{d u_{i}(t)}{d t}=-a_{i}\left(u_{i}(t)\right)\left[b_{i}\left(u_{i}(t)\right)-\sum_{j=1}^{m} p_{j i} f_{j}\left(v_{j}(t)\right)\right], \\
\frac{d v_{j}(t)}{d t}=-a_{j}\left(v_{j}(t)\right)\left[b_{j}\left(v_{j}(t)\right)-\sum_{i=1}^{n} q_{i j} g_{i}\left(u_{i}(t)\right)\right],
\end{array}\right.
$$

where $i=1,2, \cdots, n, j=1,2, \cdots, m$.

\footnotetext{
*Corresponding author

Email addresses: 2008pingc@163.com (Ping Cui), 2991726233@qq.com (Zheng-biao Li)
} 
For the sake of theoretical interest as well as application considerations, the dynamical behaviors, in particular, the existence and stability of the equilibrium point, periodic and almost periodic solutions of BAM-type Cohen-Grossberg neural networks have been extensively studied by a large number of scholars. Over the past few years, there have been considerable results on BAM-type Cohen-Grossberg neural networks (see [2, 7-14, 20, 25, 26, 28]). Recently, there have been some new results on the integral transform method (see [22-24]). In contrast, however, very few results are available on the existence and exponential stability of anti-periodic solutions for BAM-type Cohen-Grossberg neural networks, while the existence of anti-periodic solutions plays a key role in characterizing the behavior of nonlinear differential equations (see [1, 3, 4, 6, 15-19, 21, 27]).

In this paper, we consider BAM-type Cohen-Grossberg neural networks with time-varying delays described by

$$
\left\{\begin{array}{l}
\frac{d u_{i}(t)}{d t}=-a_{i}\left(u_{i}(t)\right)\left[b_{i}\left(u_{i}(t)\right)-\sum_{j=1}^{p} c_{i j} f_{i j}\left(v_{j}\left(t-\tau_{i j}\right)\right)\right], \\
\frac{d v_{j}(t)}{d t}=-d_{j}\left(v_{j}(t)\right)\left[e_{j}\left(v_{j}(t)\right)-\sum_{i=1}^{n} m_{i j} \int_{-\infty}^{t} K_{i j}(t-s) g_{i j}\left(u_{i}(s)\right) d s\right],
\end{array}\right.
$$

where $i=1,2, \cdots, n, j=1,2, \cdots, p$.

The initial conditions associated with system (1.1) are of the form

$$
\left\{\begin{array}{l}
u_{i}(\theta)=\varphi_{i}(\theta), \quad \theta \in[-\tau, 0], \quad i=1,2, \cdots, n, \\
v_{j}(\eta)=\psi_{j}(\eta), \quad \eta \in[-\infty, 0], \quad j=1,2, \cdots, p,
\end{array}\right.
$$

where $\tau=\max _{(i, j)}\left\{\tau_{i j}\right\}, \varphi_{i}$ and $\psi_{j}$ are continuous real-valued functions defined on their respective domains.

Let $x_{\mathfrak{i}}(t): R \rightarrow R$ be continuous in $t$. $x_{\mathfrak{i}}(t)$ is said to be T-anti-periodic on $R$, if $x_{\mathfrak{i}}(t+T)=-x_{\mathfrak{i}}(t)$ for all $t \in R$.

Throughout this paper, we assume that

$\left(H_{1}\right) a_{i}, d_{j}: R \rightarrow[0, \infty)$ are continuously bounded and $k_{i j}:[0, \infty) \rightarrow R$ are continuous functions and $a_{i}(-u)=-a_{i}(u), b_{i}(-u)=b_{i}(u), f_{i j}(-u)=f_{i j}(u), d_{j}(-v)=-d_{j}(v), e_{j}(-v)=e_{j}(v), u, v \in R, i=$ $1,2, \cdots, n, j=1,2, \cdots, p$;

$\left(\mathrm{H}_{2}\right) b_{i}, b_{i}^{-1}, e_{j}, e_{j}^{-1}$ are locally Lipschitz continuous and there exist positive constants $\gamma_{i}$ and $\xi_{j}$ such that

$$
b_{i}(u+x)-b_{i}(x) \geqslant \gamma_{i} u, \quad e_{j}(v+y)-e_{j}(y) \geqslant \xi_{j} v,
$$

where $u, v \in R, i=1,2, \cdots, n, j=1,2, \cdots, p ;$

$\left(\mathrm{H}_{3}\right)$ there exist constants $\lambda_{i j}>0, \mu_{i j}>0, M_{i j}>0, N_{i j}>0$ such that for all $u, v \in R, i=1,2, \cdots, n, j=$ $1,2, \cdots, p$,

$$
\begin{aligned}
& f_{i j}(0)=0, \quad\left|f_{i j}(u)-f_{i j}(v)\right| \leqslant \lambda_{i j}|u-v|, \quad\left|f_{i j}(u)\right| \leqslant M_{i j}, \\
& g_{i j}(0)=0, \quad\left|g_{i j}(u)-g_{i j}(v)\right| \leqslant \mu_{i j}|u-v|, \quad\left|g_{i j}(u)\right| \leqslant N_{i j} ;
\end{aligned}
$$

$\left(\mathrm{H}_{4}\right)$ there exists constant $\lambda>0$ such that

$$
0 \leqslant \lambda-a_{i}\left(u_{i}(t)\right)\left(\gamma_{i} e^{\lambda t}-e^{\lambda \tau} \sum_{j=1}^{p}\left|c_{i j}\right|\left|\lambda_{i j}\right|\right),
$$

and

$$
0 \leqslant \lambda-d_{j}\left(v_{j}(t)\right) \xi_{j} e^{\lambda t}-\sum_{i=1}^{n}\left|m_{i j}\right| \int_{-\infty}^{t}\left|K_{i j}(t-s)\right|\left|\mu_{i j}\right| e^{\lambda(t-s)} d s .
$$

For $x(t)=\left(u_{1}(t), u_{2}(t), \cdots, u_{n}(t), v_{1}(t), v_{2}(t), \cdots, v_{p}(t)\right)^{\top} \in R^{n+p}$, we define the norm

$$
\|x\|=\sup _{t \in R} \max \left\{\max _{1 \leqslant i \leqslant n}\left|u_{i}(t)\right|, \max _{1 \leqslant j \leqslant p}\left|v_{j}(t)\right|\right\} \text {. }
$$


Definition 1.1. Let $z^{*}(t)=\left(u_{1}^{*}(t), u_{2}^{*}(t), \cdots, u_{n}^{*}(t), v_{1}^{*}(t), v_{2}^{*}(t), \cdots, v_{p}^{*}(t)\right)^{\top}$ be an anti-periodic solution of system (1.1) with initial value $\left(\varphi_{1}^{*}(t), \varphi_{2}^{*}(t), \cdots, \varphi_{n}^{*}(t), \psi_{1}^{*}(t), \psi_{2}^{*}(t), \cdots, \psi_{p}^{*}(t)\right)^{\top}$. If there exist constants $\lambda>0$ and $M>1$ such that for every solution $z(t)=\left(u_{1}(t), u_{2}(t), \cdots, u_{n}(t), v_{1}(t), v_{2}(t), \cdots, v_{p}(t)\right)^{\top}$ of system (1.1) with initial value $\left(\varphi_{1}(t), \varphi_{2}(t), \cdots, \varphi_{n}(t), \psi_{1}(t), \psi_{2}(t), \cdots, \psi_{p}(t)\right)^{\top}$ satisfies

$$
\left\|z-z^{*}\right\| \leqslant M e^{-\lambda t} \max \left\{\left\|\varphi-\varphi^{*}\right\|_{\infty}, \quad\left\|\psi-\psi^{*}\right\|_{\infty}\right\}, \quad t>0, \quad i=1,2, \cdots, n, j=1,2, \cdots, p .
$$

where

$$
\left\|\varphi-\varphi^{*}\right\|_{\infty}=\sup _{-\tau \leqslant s \leqslant 0} \max _{1 \leqslant i \leqslant n}\left|\varphi_{i}(s)-\varphi_{i}^{*}(s)\right|, \quad\left\|\psi-\psi^{*}\right\|_{\infty}=\sup _{-\infty \leqslant s \leqslant 0} \max _{1 \leqslant j \leqslant p}\left|\psi_{i}(s)-\psi_{i}^{*}(s)\right|,
$$

then $z^{*}(t)$ is said to be globally exponentially stable.

\section{Preliminaries}

The following lemmas will be used to prove our main results in Section 3.

Lemma 2.1. Let $\left(\mathrm{H}_{1}\right)-\left(\mathrm{H}_{4}\right)$ hold. Suppose that $\tilde{z}(\mathrm{t})=(\tilde{\mathrm{u}}(\mathrm{t}), \tilde{v}(\mathrm{t}))$, where $\tilde{\mathfrak{u}}(\mathrm{t})=\left(\tilde{u}_{1}(\mathrm{t}), \tilde{u}_{2}(\mathrm{t}), \cdots, \tilde{u}_{\mathfrak{n}}(\mathrm{t})\right)^{\top}$, $\tilde{v}(t)=\left(\tilde{v}_{1}(t), \tilde{v}_{2}(t), \cdots, \tilde{v}_{p}(t)\right)^{\top}$ is a solution of system (1.1) with initial conditions

$$
\begin{gathered}
\tilde{u}_{i}(\theta)=\tilde{\varphi}_{i}(\theta), \quad\left|\tilde{\varphi}_{i}(\theta)\right|<\frac{\sum_{j=1}^{p}\left|c_{i j}\right| M_{i j}}{\gamma_{i}}, \quad \theta \in[-\tau, 0], \quad i=1,2, \ldots, n, \\
\tilde{v}_{j}(\eta)=\tilde{\psi}_{j}(\eta), \quad\left|\tilde{\psi}_{j}(\eta)\right|<\frac{\sum_{i=1}^{n}\left|m_{i j}\right| N_{i j} \int_{-\infty}^{\rho}\left|K_{i j}(\rho-s)\right| d s}{\xi_{j}}, \quad \eta \in(-\infty, 0], \quad j=1,2, \ldots, p .
\end{gathered}
$$

Then

$$
\left|\tilde{u}_{i}(t)\right|<\frac{\sum_{j=1}^{p}\left|c_{i j}\right| M i j}{\gamma_{i}},\left|\tilde{v}_{j}(t)\right|<\frac{\sum_{i=1}^{n}\left|m_{i j}\right| N i j \int_{-\infty}^{\rho}\left|K_{i j}(\rho-s)\right| d s}{\xi_{j}},
$$

where $t \geqslant 0, i=1,2, \cdots, n, j=1,2, \cdots, p$.

Proof. By way of contradiction, assume that (2.3) does not hold. Then, there exists $\rho>0$ such that

$$
\begin{gathered}
\tilde{u}_{i}(\rho)=\frac{\sum_{j=1}^{p}\left|c_{i j}\right| M_{i j}}{\gamma_{i}}, \quad \tilde{u}_{i}(t)<\frac{\sum_{j=1}^{p}\left|c_{i j}\right| M_{i j}}{\gamma_{i}}, t \in[-\tau, \rho], \\
\tilde{v}_{j}(\rho)=\frac{\sum_{i=1}^{n}\left|m_{i j}\right| N_{i j} \int_{-\infty}^{\rho}\left|K_{i j}(\rho-s)\right| d s}{\xi_{j}}, \quad \tilde{v}_{j}(t)<\frac{\sum_{i=1}^{n}\left|m_{i j}\right| N_{i j} \int_{-\infty}^{\rho}\left|K_{i j}(\rho-s)\right| d s}{\xi_{j}}, t \in[-\infty, \rho] .
\end{gathered}
$$

Calculating the upper left derivative of $\left|\tilde{u}_{i}(t)\right|$ and $\left|\tilde{v}_{j}(t)\right|$, together with $\left(\mathrm{H}_{1}\right)-\left(\mathrm{H}_{4}\right),(2.4)$ and (2.5), we can obtain

$$
\begin{aligned}
0 & \leqslant D^{+}\left(\left|\tilde{u}_{i}(\rho)\right|\right) \\
& \leqslant-a_{i}\left(\tilde{u}_{i}(\rho)\right) b_{i}\left(\tilde{u}_{i}(\rho)\right)+a_{i}\left(\tilde{u}_{i}(\rho)\right)\left|\sum_{j=1}^{p} c_{i j} f_{i j}\left(\tilde{v}_{j}\left(\rho-\tau_{i j}\right)\right)\right|
\end{aligned}
$$




$$
\begin{aligned}
& \leqslant a_{i}\left(\tilde{u}_{i}(\rho)\right)\left[-b_{i}\left(\tilde{u}_{i}(\rho)\right)+\sum_{j=1}^{p}\left|c_{i j}\right|\left|f_{i j}\left(\tilde{v}_{j}\left(\rho-\tau_{i j}\right)\right)\right|\right] \\
& \leqslant a_{i}\left(\tilde{u}_{i}(\rho)\right)\left[\sum_{j=1}^{p}\left|c_{i j}\right| M_{i j}-b_{i}\left(\tilde{u}_{i}(\rho)\right)\right] \\
& \leqslant a_{i}\left(\tilde{u}_{i}(\rho)\right)\left[\sum_{j=1}^{p}\left|c_{i j}\right| M_{i j}-\gamma_{i} \tilde{u}_{i}(\rho)\right] \\
& <0
\end{aligned}
$$

and

$$
\begin{aligned}
0 & \leqslant \mathrm{D}^{+}\left(\left|\tilde{v}_{j}(\rho)\right|\right) \\
& \leqslant-\mathrm{d}_{j}\left(\tilde{v}_{j}(\rho)\right) e_{j}\left(\tilde{v}_{j}(\rho)\right)+\mathrm{d}_{j}\left(\tilde{v}_{j}(\rho)\right)\left|\sum_{i=1}^{n} m_{i j} \int_{-\infty}^{\rho} K_{i j}(\rho-s) g_{i j}\left(\tilde{u}_{i}(s)\right) d s\right| \\
& \leqslant d_{j}\left(\tilde{v}_{j}(\rho)\right)\left[\sum_{i=1}^{n}\left|m_{i j}\right| \int_{-\infty}^{\rho}\left|K_{i j}(\rho-s)\right|\left|g_{i j}\left(\tilde{u}_{i}(s)\right)\right| d s-e_{j}\left(\tilde{v}_{j}(\rho)\right)\right] \\
& \leqslant d_{j}\left(\tilde{v}_{j}(\rho)\right)\left[\sum_{i=1}^{n}\left|m_{i j}\right| N_{i j} \int_{-\infty}^{\rho}\left|K_{i j}(\rho-s)\right| d s-\xi_{j} \tilde{v}_{j}(\rho)\right] \\
& <0,
\end{aligned}
$$

which is a contradiction and hence (2.3) holds. This completes the proof.

Remark 2.2. In view of the boundedness of this solution, from the theory of functional differential equations in [1], it follows that $\tilde{u}(t)$ can be defined on $[-\tau, \infty)$ and $\tilde{v}(t)$ can be defined on $[0, \infty)$.

Lemma 2.3. Suppose that $\left(\mathrm{H}_{1}\right)-\left(\mathrm{H}_{4}\right)$ are satisfied. Let $z^{*}(\mathrm{t})=\left(\mathrm{u}^{*}(\mathrm{t}), v^{*}(\mathrm{t})\right)^{\mathrm{T}}$, where $\mathrm{u}^{*}(\mathrm{t})=\left(\mathrm{u}_{1}^{*}(\mathrm{t}), \mathrm{u}_{2}^{*}(\mathrm{t}), \cdots\right.$, $\left.\mathrm{u}_{\mathrm{n}}^{*}(\mathrm{t})\right), v^{*}(\mathrm{t})=\left(v_{1}^{*}(\mathrm{t}), v_{2}^{*}(\mathrm{t}), \cdots, \mathrm{u}_{\mathrm{p}}^{*}(\mathrm{t})\right)$ be the solution of system (1.1) with initial value (2.1) and (2.2). Let $z(t)=\left(u_{1}(t), u_{2}(t), \cdots, u_{n}(t), v_{1}(t), v_{2}(t), \cdots, u_{p}(t)\right)^{\top}$ be the solution of system (1.1) with initial value $\left(\varphi_{1}(t), \varphi_{2}(t), \cdots, \varphi_{n}(t), \psi_{1}(t), \psi_{2}(t), \cdots, \psi_{p}(t)\right)^{\top}$. Then there exist constants $\lambda>0$ and $M>1$ such that

$$
\left\|z-z^{*}\right\| \leqslant M e^{-\lambda t} \max \left\{\left\|\varphi-\varphi^{*}\right\|_{\infty},\left\|\psi-\psi^{*}\right\|_{\infty}\right\}, \quad t>0 .
$$

Proof. Set $x(t)=u(t)-u^{*}(t)$ and $y(t)=v(t)-v^{*}(t)$, by system (1.1), we have

$$
\left\{\begin{aligned}
\frac{d x_{i}(t)}{d t}= & -a_{i}\left(x_{i}(t)+u_{i}^{*}(t)\right)\left[b_{i}\left(x_{i}(t)+u_{i}^{*}(t)\right)-b_{i}\left(u_{i}^{*}(t)\right)\right. \\
& \left.-\sum_{j=1}^{p} c_{i j}\left(f_{i j}\left(y_{j}\left(t-\tau_{i j}\right)+v_{j}^{*}\left(t-\tau_{i j}\right)\right)-f_{i j}\left(v_{j}^{*}\left(t-\tau_{i j}\right)\right)\right)\right], i=1,2, \cdots, n, \\
\frac{d y_{j}(t)}{d t}= & -d_{j}\left(y_{j}(t)+v_{j}^{*}(t)\right)\left[e_{j}\left(y_{j}(t)+v_{j}^{*}(t)\right)-e_{j}\left(v_{j}^{*}(t)\right)\right. \\
& \left.-\sum_{i=1}^{n} m_{i j} \int_{-\infty}^{t} K_{i j}(t-s)\left(g_{i j}\left(x_{i}(s)+u_{i}^{*}(t)\right)-g_{i j}\left(u_{i}^{*}(t)\right)\right) d s\right], \quad j=1,2, \cdots, p .
\end{aligned}\right.
$$

We consider the Lyapunov functional

$$
V_{i}^{\{1\}}(t)=\left|x_{i}(t)\right| e^{\lambda t}, \quad V_{j}^{\{2\}}(t)=\left|y_{j}(t)\right| e^{\lambda t}, \quad i=1,2, \cdots, n, j=1,2, \cdots, p .
$$


Calculating the upper right derivative of $V_{i}^{\{1\}}(t)$, we have

$$
\begin{aligned}
D^{+}\left(V_{i}^{\{1\}}(t)\right) \leqslant & -a_{i}\left(\left|x_{i}(t)\right| e^{\lambda t}+u_{i}^{*}(t)\right)\left[b_{i}\left(\left|x_{i}(t)\right| e^{\lambda t}+u_{i}^{*}(t)\right)-b_{i}\left(u_{i}^{*}(t)\right)\right. \\
& \left.-\sum_{j=1}^{p}\left|c_{i j}\right|\left|f_{i j}\left(y_{j}\left(t-\tau_{i j}\right)+v_{j}^{*}\left(t-\tau_{i j}\right)\right)-f_{i j}\left(v_{j}^{*}\left(t-\tau_{i j}\right)\right)\right|\right] e^{\lambda t}+\lambda\left|x_{i}(t)\right| e^{\lambda t} \\
\leqslant & -a_{i}\left(\left|x_{i}(t)\right| e^{\lambda t}+u_{i}^{*}(t)\right)\left[\gamma_{i}\left|x_{i}(t)\right| e^{\lambda t}\right. \\
& \left.-\sum_{j=1}^{p}\left|c_{i j}\right|\left|\lambda_{i j}\right|\left|y_{j}\left(t-\tau_{i j}\right)\right|\right] e^{\lambda t}+\lambda\left|x_{i}(t)\right| e^{\lambda t} \\
= & a_{i}\left(\left|x_{i}(t)\right| e^{\lambda t}+u_{i}^{*}(t)\right) \sum_{j=1}^{p}\left|c_{i j}\right|\left|\lambda_{i j}\right|\left|y_{j}\left(t-\tau_{i j}\right)\right| e^{\lambda\left(t-\tau_{i j}\right)} e^{\lambda \tau_{i j}} \\
& +\left[\lambda-a_{i}\left(\left|x_{i}(t)\right| e^{\lambda t}+u_{i}^{*}(t)\right) \gamma_{i} e^{\lambda t}\right]\left|x_{i}(t)\right| e^{\lambda t} .
\end{aligned}
$$

Calculating the upper right derivative of $V_{j}^{\{2\}}(t)$, we have

$$
\begin{aligned}
D^{+}\left(v_{j}^{\{2\}}(t)\right) \leqslant & -d_{j}\left(\left|y_{j}(t)\right| e^{\lambda t}+v_{j}^{*}(t)\right)\left[e_{j}\left(\left|y_{j}(t)\right| e^{\lambda t}+v_{j}^{*}(t)\right)-e_{j}\left(v_{j}^{*}(t)\right)\right. \\
& \left.-\sum_{i=1}^{n}\left|m_{i j}\right| \int_{-\infty}^{t}\left|K_{i j}(t-s)\right|\left|g_{i j}\left(x_{i}(s)+u_{i}^{*}(t)\right)-g_{i j}\left(u_{i}^{*}(t)\right)\right| d s\right] e^{\lambda t}+\lambda\left|y_{j}(t)\right| e^{\lambda t} \\
\leqslant & -d_{j}\left(\left|y_{j}(t)\right| e^{\lambda t}+v_{j}^{*}(t)\right)\left[\xi_{j}\left|y_{j}(t)\right| e^{\lambda t}\right. \\
& \left.-\sum_{i=1}^{n}\left|m_{i j}\right| \int_{-\infty}^{t}\left|K_{i j}(t-s)\right|\left|\mu_{i j}\right|\left|x_{i}(s)\right| d s\right] e^{\lambda t}+\lambda\left|y_{j}(t)\right| e^{\lambda t} \\
\leqslant & {\left[\lambda-d_{j}\left(\left|y_{j}(t)\right| e^{\lambda t}+v_{j}^{*}(t)\right) \xi_{j} e^{\lambda t}\right]\left|y_{j}(t)\right| e^{\lambda t}+d_{j}\left(\left|y_{j}(t)\right| e^{\lambda t}+v_{j}^{*}(t)\right) } \\
& \times \sum_{i=1}^{n}\left|m_{i j}\right| \int_{-\infty}^{t}\left|K_{i j}(t-s)\right|\left|\mu_{i j}\right|\left|x_{i}(s)\right| e^{\lambda s} e^{\lambda(t-s)} d s .
\end{aligned}
$$

Let $M>1$ denote an arbitrary real number and set

$$
\begin{aligned}
\left\|\varphi-\varphi^{*}\right\|_{\infty} & =\sup _{-\tau \leqslant s \leqslant 0} \max _{1 \leqslant i \leqslant n}\left|\varphi_{i}(s)-\varphi_{i}^{*}(s)\right|>0, \\
\left\|\psi-\psi^{*}\right\|_{\infty} & =\sup _{-\infty \leqslant s \leqslant 0} \max _{1 \leqslant j \leqslant p}\left|\psi_{i}(s)-\psi_{i}^{*}(s)\right|>0 .
\end{aligned}
$$

It follows from (2.6) that

$$
V_{i}^{\{1\}}(t)=\left|x_{i}(t)\right| e^{\lambda t}<M\left\|\varphi-\varphi^{*}\right\|_{\infty}, \quad V_{j}^{\{2\}}(t)=\left|y_{j}(t)\right| e^{\lambda t}<M\left\|\psi-\psi^{*}\right\|_{\infty},
$$

for all $t \in(-\infty, 0], i=1,2, \cdots, n, j=1,2, \cdots, p$.

We claim that

$$
V_{i}^{\{1\}}(t)=\left|x_{i}(t)\right| e^{\lambda t}<M\left\|\varphi-\varphi^{*}\right\|_{\infty}, \quad V_{j}^{\{2\}}(t)=\left|y_{j}(t)\right| e^{\lambda t}<M\left\|\psi-\psi^{*}\right\|_{\infty},
$$

for all $t>0, i=1,2, \cdots, n, j=1,2, \cdots, p$. Contrarily, there must exist $i \in\{1,2, \cdots, n\}, j \in\{1,2, \cdots, p\}$ and $t_{i}>0, t_{j}>0$ such that

$$
V_{i}^{\{1\}}\left(t_{i}\right)=M\left\|\varphi-\varphi^{*}\right\|_{\infty}, \quad V_{\bar{i}}^{\{1\}}(t)<M\left\|\varphi-\varphi^{*}\right\|_{\infty}, \forall t \in\left(-\infty, t_{i}\right),
$$




$$
V_{j}^{\{2\}}\left(t_{j}\right)=M\left\|\psi-\psi^{*}\right\|_{\infty}, \quad V_{\bar{j}}^{\{2\}}(t)<M\left\|\psi-\psi^{*}\right\|_{\infty}, \forall t \in\left(-\infty, t_{j}\right),
$$

where $\bar{i} \in\{1,2, \cdots, n\}, \bar{j} \in\{1,2, \cdots, p\}$, which is

$$
\begin{aligned}
& V_{i}^{\{1\}}\left(t_{i}\right)-M\left\|\varphi-\varphi^{*}\right\|_{\infty}=0, \quad V_{\bar{i}}^{\{1\}}(t)-M\left\|\varphi-\varphi^{*}\right\|_{\infty}<0, \quad \forall t \in\left(-\infty, t_{i}\right), \\
& V_{j}^{\{2\}}\left(t_{j}\right)-M\left\|\psi-\psi^{*}\right\|_{\infty}=0, \quad V_{\bar{j}}^{\{2\}}(t)-M\left\|\psi-\psi^{*}\right\|_{\infty}<0, \quad \forall t \in\left(-\infty, t_{j}\right),
\end{aligned}
$$

where $\bar{i} \in\{1,2, \cdots, n\}, \bar{j} \in\{1,2, \cdots, p\}$. Together with (2.6), (2.7), (2.8), (2.9), we obtain

$$
\begin{aligned}
0 \leqslant & D^{+}\left(V_{i}^{\{1\}}\left(t_{i}\right)-M\left\|\varphi-\varphi^{*}\right\|\right) \\
= & D^{+}\left(V_{i}^{\{1\}}\left(t_{i}\right)\right) \\
\leqslant & {\left[\lambda-a_{i}\left(\left|x_{i}\left(t_{i}\right)\right| e^{\lambda t_{i}}+u_{i}^{*}\left(t_{i}\right)\right) \gamma_{i} e^{\lambda t_{i}}\right]\left|x_{i}\left(t_{i}\right)\right| e^{\lambda} t_{i} } \\
& +a_{i}\left(\left|x_{i}\left(t_{i}\right)\right| e^{\lambda t_{i}}+u_{i}^{*}\left(t_{i}\right)\right) \sum_{j=1}^{p}\left|c_{i j}\right|\left|\lambda_{i j}\right|\left|y_{j}\left(t_{i}-\tau_{i j}\right)\right| e^{\lambda\left(t_{i}-\tau_{i j}\right)} e^{\lambda \tau_{i j}} \\
\leqslant & {\left[\lambda-a_{i}\left(\left|x_{i}\left(t_{i}\right)\right| e^{\lambda t_{i}}+u_{i}^{*}\left(t_{i}\right)\right) \gamma_{i} e^{\lambda t_{i}}\right] M\left\|\varphi-\varphi^{*}\right\|_{\infty} } \\
& +a_{i}\left(\left|x_{i}\left(t_{i}\right)\right| e^{\lambda t_{i}}+u_{i}^{*}\left(t_{i}\right)\right) \sum_{j=1}^{p}\left|c_{i j}\right|\left|\lambda_{i j}\right| M\left\|\psi-\psi^{*}\right\|_{\infty} e^{\lambda \tau_{i j}} \\
\leqslant & {\left[\lambda-a_{i}\left(\left|x_{i}\left(t_{i}\right)\right| e^{\lambda t_{i}}+u_{i}^{*}\left(t_{i}\right)\right)\left(\gamma_{i} e^{\lambda t_{i}}-e^{\lambda \tau} \sum_{j=1}^{p}\left|c_{i j} \| \lambda_{i j}\right|\right)\right] } \\
& \times \max \left\{M\left\|\varphi-\varphi^{*}\right\|_{\infty}, M\left\|\psi-\psi^{*}\right\|_{\infty}\right\},
\end{aligned}
$$

and

$$
\begin{aligned}
0 \leqslant & D^{+}\left(V_{j}^{\{2\}}\left(t_{j}\right)-M\left\|\psi-\psi^{*}\right\|\right) \\
= & D^{+}\left(V_{j}^{\{2\}}\left(t_{j}\right)\right) \\
\leqslant & {\left[\lambda-d_{j}\left(\left|y_{j}\left(t_{j}\right)\right| e^{\lambda t_{j}}+v_{j}^{*}\left(t_{j}\right)\right) \xi_{j} e^{\lambda t_{j}}\right]\left|y_{j}\left(t_{j}\right)\right| e^{\lambda t_{j}}+d_{j}\left(\left|y_{j}\left(t_{j}\right)\right| e^{\lambda t_{j}}\right.} \\
& \left.+v_{j}^{*}\left(t_{j}\right)\right) \times \sum_{i=1}^{n}\left|m_{i j}\right| \int_{-\infty}^{t_{j}}\left|K_{i j}\left(t_{j}-s\right)\right|\left|\mu_{i j}\right|\left|x_{i}(s)\right| e^{\lambda s} e^{\lambda\left(t_{j}-s\right)} d s \\
\leqslant & {\left[\lambda-d_{j}\left(\left|y_{j}\left(t_{j}\right)\right| e^{\lambda t_{j}}+v_{j}^{*}\left(t_{j}\right)\right) \xi_{j} e^{\lambda t_{j}}\right] M\left\|\psi-\psi^{*}\right\|_{\infty}+d_{j}\left(\left|y_{j}\left(t_{j}\right)\right| e^{\lambda t_{j}}\right.} \\
& \left.+v_{j}^{*}\left(t_{j}\right)\right) \times \sum_{i=1}^{n}\left|m_{i j}\right| \int_{-\infty}^{t_{j}}\left|K_{i j}\left(t_{j}-s\right)\right|\left|\mu_{i j}\right| e^{\lambda\left(t_{j}-s\right)} M\left\|\varphi-\varphi^{*}\right\|_{\infty} d s \\
\leqslant & {\left[\lambda-d_{j}\left(\left|y_{j}\left(t_{j}\right)\right| e^{\lambda t_{j}}+v_{j}^{*}\left(t_{j}\right)\right)\right.} \\
& \left.\times\left(\xi_{j} e^{\lambda t_{j}}-\sum_{i=1}^{n}\left|m_{i j}\right| \int_{-\infty}^{t_{j}}\left|K_{i j}\left(t_{j}-s\right)\right|\left|\mu_{i j}\right| e^{\lambda\left(t_{j}-s\right)}\right) d s\right] \\
& \times \max \left\{M\left\|\varphi-\varphi^{*}\right\|_{\infty}, M\left\|\psi-\psi^{*}\right\|_{\infty}\right\} .
\end{aligned}
$$

Thus

$$
0 \leqslant \lambda-a_{i}\left(\left|x_{i}\left(t_{i}\right)\right| e^{\lambda t_{i}}+u_{i}^{*}\left(t_{i}\right)\right)\left(\gamma_{i} e^{\lambda t_{i}}-e^{\lambda \tau} \sum_{j=1}^{p}\left|c_{i j}\right|\left|\lambda_{i j}\right|\right),
$$


and

$$
0 \leqslant \lambda-d_{j}\left(\left|y_{j}\left(t_{j}\right)\right| e^{\lambda t_{j}}+v_{j}^{*}\left(t_{j}\right)\right)\left(\xi_{j} e^{\lambda t_{j}}-\sum_{i=1}^{n}\left|m_{i j}\right| \int_{-\infty}^{t_{j}}\left|K_{i j}\left(t_{j}-s\right)\right|\left|\mu_{i j}\right| e^{\lambda\left(t_{j}-s\right)} d s\right),
$$

which is a contradiction. Hence, (2.10) holds. It follows that

$$
\left|x_{i}(t)\right|<M\left\|\varphi-\varphi^{*}\right\|_{\infty} e^{-\lambda t}, \quad\left|y_{j}(t)\right|<M\left\|\psi-\psi^{*}\right\|_{\infty} e^{-\lambda t}, \quad t>0, \quad i=1,2, \cdots, n, \quad j=1,2, \cdots, p .
$$

This completes the proof of Lemma 2.3 .

If $z^{*}(t)=\left(u^{*}(t), v^{*}(t)\right)^{\top}$, where $\mathrm{u}^{*}(\mathrm{t})=\left(\mathrm{u}_{1}^{*}(\mathrm{t}), \cdots, \mathrm{u}_{\mathfrak{n}}^{*}(\mathrm{t})\right), v^{*}(\mathrm{t})=\left(v_{1}^{*}(\mathrm{t}), \cdots, \mathrm{u}_{\mathrm{p}}^{*}(\mathrm{t})\right)$ is a T-antiperiodic solution of system (1.1), it follows from Lemma 2.3 and Definition 1.1 that $z^{*}(t)$ is globally exponentially stable.

\section{Main results}

Our main result of this paper is as follows.

Theorem 3.1. Suppose that $\left(\mathrm{H}_{1}\right)-\left(\mathrm{H}_{4}\right)$ are satisfied. Then system (1.1) has exactly one T-anti-periodic solution $z^{*}(t)$. Moreover, $z^{*}(t)$ is globally exponentially stable.

Proof. Let $z(t)=(u(t), v(t))$, where $u(t)=\left(u_{1}(t), u_{2}(t), \cdots, u_{n}(t)\right)^{\top}, v(t)=\left(v_{1}(t), v_{2}(t), \cdots, v_{p}(t)\right)^{\top}$ be a solution of system (1.1) with initial conditions

$$
\begin{aligned}
& u_{i}(\theta)=\varphi_{i}^{v}(\theta),\left|\varphi_{i}^{v}(\theta)\right|<\frac{\sum_{j=1}^{p}\left|c_{i j}\right| M_{i j}}{\gamma_{i}}, \quad \theta \in[-\tau, 0], \quad i=1,2, \cdots, n, \\
& v_{j}(\eta)=\psi_{j}^{v}(\eta),\left|\psi_{j}^{v}(\eta)\right|<\frac{\sum_{i=1}^{n}\left|m_{i j}\right| N_{i j} \int_{-\infty}^{\rho}\left|K_{i j}(\rho-s)\right| d s}{\xi_{j}}, \quad \eta \in(-\infty, 0], j=1,2, \cdots, p .
\end{aligned}
$$

By Lemma 2.1, the solution $z(t)=(u(t), v(t))$ is bounded and

$$
\begin{aligned}
& \left|u_{i}(t)\right|<\frac{\sum_{j=1}^{p}\left|c_{i j}\right| M_{i j}}{\gamma_{i}}, \quad t \in[-\tau, 0], \quad i=1,2, \cdots, n, \\
& \left|v_{j}(t)\right|<\frac{\sum_{i=1}^{n}\left|m_{i j}\right| N_{i j} \int_{-\infty}^{\rho}\left|K_{i j}(\rho-s)\right| d s}{\xi_{j}}, \quad t \in(-\infty, 0], j=1,2, \cdots, p .
\end{aligned}
$$

From (1.1) and $\left(\mathrm{H}_{1}\right)-\left(\mathrm{H}_{4}\right)$, we have

$$
\begin{aligned}
\left((-1)^{k+1} u_{i}(t+(k+1) T)\right)^{\prime}= & (-1)^{k+1} u_{i}^{\prime}(t+(k+1) T) \\
= & (-1)^{k+1}\left\{-a_{i}\left(u_{i}(t+(k+1) T)\right)\left[b_{i}\left(u_{i}(t+(k+1) T)\right)\right.\right. \\
& \left.\left.-\sum_{j=1}^{p} c_{i j} f_{i j}\left(v_{j}\left((t+(k+1) T)-\tau_{i j}\right)\right)\right]\right\} \\
= & -a_{i}\left((-1)^{k+1} u_{i}(t+(k+1) T)\right)\left[b_{i}\left((-1)^{k+1} u_{i}(t+(k+1) T)\right)\right. \\
& \left.-\sum_{j=1}^{p} c_{i j} f_{i j}\left((-1)^{k+1} v_{j}\left((t+(k+1) T)-\tau_{i j}\right)\right)\right], \quad i=1,2, \cdots, n,
\end{aligned}
$$


and

$$
\begin{aligned}
\left((-1)^{k+1} v_{j}(t+(k+1) T)\right)^{\prime}= & (-1)^{k+1} v_{j}^{\prime}(t+(k+1) T) \\
= & (-1)^{k+1}\left\{-d_{j}\left(v_{j}(t+(k+1) T)\right)\left[e_{j}\left(v_{j}(t+(k+1) T)\right)\right.\right. \\
& \left.\left.-\sum_{i=1}^{n} m_{i j} \int_{-\infty}^{t+(k+1) T} k_{i j}(t+(k+1) T-s) g_{i j}\left(u_{i}(s)\right) d s\right]\right\} \\
= & -d_{j}\left(v_{j}(t+(k+1) T)\right)\left[e_{j}\left((-1)^{k+1} v_{j}(t+(k+1) T)\right)\right. \\
& \left.-\sum_{i=1}^{n} m_{i j} \int_{-\infty}^{t+(k+1) T} k_{i j}(t+(k+1) T-s) g_{i j}\left(u_{i}(s)\right) d s\right], j=1,2, \cdots, p .
\end{aligned}
$$

Thus, for any natural number $k,(-1)^{k+1} z(t+(k+1) T)$ are the solution of system (1.1). Then, by Lemma 2.3 , there exists a constant $M>0$ such that

$$
\begin{aligned}
\mid(-1)^{k+1} u_{i}(t+(k+1) T) & -(-1)^{k} u_{i}(t+k T) \mid \\
& \leqslant M e^{-\lambda(t+k T)} \sup _{-\tau \leqslant s \leqslant 0} \max _{1 \leqslant i \leqslant n}\left|u_{i}(s+T)+u_{i}(s)\right| \\
& \leqslant 2 e^{-\lambda(t+k T)} M \frac{\sum_{j=1}^{p}\left|c_{i j}\right| M_{i j}}{\gamma_{i}}, \text { for } t+k T>0, i=1,2, \cdots, n,
\end{aligned}
$$

and

$$
\begin{aligned}
\mid(-1)^{k+1} v_{j}(t+ & (k+1) T)-(-1)^{k} v_{j}(t+k T) \mid \\
& \leqslant M e^{-\lambda(t+k T)} \sup _{-\infty \leqslant s \leqslant 0} \max _{1 \leqslant j \leqslant p}\left|v_{j}(s+T)+v_{j}(s)\right| \\
& \leqslant 2 e^{-\lambda(t+k T)} M \frac{\sum_{i=1}^{n}\left|m_{i j}\right| N_{i j} \int_{-\infty}^{\rho}\left|K_{i j}(\rho-s)\right| d s}{\xi_{j}}, \text { for } t+k T>0, j=1,2, \ldots, p .
\end{aligned}
$$

Thus, for any natural number $m$, we obtain

$$
(-1)^{m+1} u_{i}(t+(m+1) T)=u_{i}(t)+\sum_{k=0}^{m}\left[(-1)^{k+1} u_{i}(t+(k+1) T)-(-1)^{k} u_{i}(t+k T)\right],
$$

and

$$
(-1)^{m+1} v_{j}(t+(m+1) T)=v_{j}(t)+\sum_{k=0}^{m}\left[(-1)^{k+1} v_{j}(t+(k+1) T)-(-1)^{k} v_{j}(t+k T)\right],
$$

where $i=1,2, \cdots, n, j=1,2, \cdots, p$. Then,

$$
\left|(-1)^{m+1} u_{i}(t+(m+1) T)\right| \leqslant\left|u_{i}(t)\right|+\sum_{k=0}^{m}\left|(-1)^{k+1} u_{i}(t+(k+1) T)-(-1)^{k} u_{i}(t+k T)\right|,
$$

and

$$
\left|(-1)^{m+1} v_{j}(t+(m+1) T)\right| \leqslant\left|v_{j}(t)\right|+\sum_{k=0}^{m}\left|(-1)^{k+1} v_{j}(t+(k+1) T)-(-1)^{k} v_{j}(t+k T)\right|,
$$

where $i=1,2, \cdots, n, j=1,2, \cdots, p$.

In view of (3.1) and (3.2), we can choose sufficiently large constants $N_{1}>0, N_{2}>0$ and positive constants $\alpha_{1}, \alpha_{2}$ such that 


$$
\left|(-1)^{k+1} u_{i}(t+(k+1) T)-(-1)^{k} u_{i}(t+k T)\right| \leqslant \alpha_{1}\left(e^{-\lambda t}\right)^{k}, \quad \text { for } k>N_{1}, \quad i=1, \cdots, n,
$$

and

$$
\left|(-1)^{k+1} v_{j}(t+(k+1) T)-(-1)^{k} v_{j}(t+k T)\right| \leqslant \alpha_{2}\left(e^{-\lambda t}\right)^{k}, \quad \text { for } k>N_{2}, \quad j=1, \ldots, p .
$$

It follows from above that $\left\{(-1)^{m} z(t+m T)\right\}$ uniformly converges to a continuous function $z^{*}(t)=$ $\left(u^{*}(t), v^{*}(t)\right)^{\top}$, where $u^{*}(t)=\left(u_{1}^{*}(t), u_{2}^{*}(t), \cdots, u_{n}^{*}(t)\right), v^{*}(t)=\left(v_{1}^{*}(t), v_{2}^{*}(t), \cdots, v_{p}^{*}(t)\right)$ on any compact set of $R$.

Now we will show that $z^{*}(t)$ is T-anti-periodic solution of system (1.1). First, $z^{*}(t)$ is T-anti-periodic, since

$$
z^{*}(t+T)=\lim _{m \rightarrow \infty}(-1)^{m} z(t+T+m T)=-\lim _{(m+1) \rightarrow \infty}(-1)^{m+1} z(t+(m+1) T)=-z^{*}(t) .
$$

Next, we prove that $z^{*}(t)$ is a solution of (1.1). In fact, together with the continuity of the right side of (1.1), (3.1) implies that $\left\{\left((-1)^{m+1} z(t+(m+1) T)\right)^{\prime}\right\}$ uniformly converges to a continuous function on any compact set of $R$. Thus, letting $m \rightarrow \infty$, we obtain

$$
\frac{d}{d t}\left\{u_{i}^{*}(t)\right\}=-a_{i}\left(u_{i}^{*}(t)\right)\left[b_{i}\left(u_{i}^{*}(t)\right)-\sum_{j=1}^{p} c_{i j} f_{i j}\left(v_{j}\left(t-\tau_{i j}\right)\right)\right],
$$

and

$$
\frac{d}{d t}\left\{v_{j}^{*}(t)\right\}=-d_{j}\left(v_{j}^{*}(t)\right)\left[e_{j}\left(v_{j}^{*}(t)\right)-\sum_{i=1}^{n} m_{i j} \int_{-\infty}^{t} K_{i j}(t-s) g_{i j}\left(u_{i}(s)\right) d s\right] .
$$

Therefore, $z^{*}(t)$ is a solution of (1.1).

Finally, by Lemma 2.3 , we can prove that $z^{*}(t)$ is globally exponentially stable. This completes the proof.

\section{Acknowledgment}

This work is supported by the Natural Science Foundation of the People's Republic of China (Grant No.51463021,11361048) and the Education Department Foundation of Yunnan Province (08C0185).

\section{References}

[1] S. Aizicovici, M. McKibben, S. Reich, Anti-periodic solutions to nonmonotone evolution equations with discontinuous nonlinearities, Nonlinear Anal., 43 (2001), 233-251. 1, 2.2

[2] C.-Z. Bai, Stability analysis of Cohen-Grossberg BAM neural networks with delays and impulses, Chaos Solitons Fractals, 35 (2008), 263-267. 1

[3] Y.-Q. Chen, Anti-periodic solutions for semilinear evolution equations, J. Math. Anal. Appl., 315 (2006), 337-348. 1

[4] Y.-Q. Chen, J. J. Nieto, D. O'Regan, Anti-periodic solutions for fully nonlinear first-order differential equations, Math. Comput. Modelling, 46 (2007), 1183-1190. 1

[5] M. A. Cohen, S. Grossberg, Absolute stability of global pattern formation and parallel memory storage by competitive neural networks, IEEE Trans. Systems Man Cybernet., 13 (1983), 815-826. 1

[6] C.-H. Feng, R. Plamondon, Stability analysis of bidirectional associative memory networks with time delays, IEEE Trans. Neural Netw., 14 (2003), 1560-1565. 1

[7] K. Gopalsamy, X.-Z. He, Delay-independent stability in bidirectional associative memory networks, IEEE Trans. Neural Netw., 5 (1994), 998-1002. 1

[8] J. Hale, Theory of functional differential equations, Second edition, Applied Mathematical Sciences, Springer-Verlag, New York-Heidelberg, (1977)

[9] J. J. Hopfield, Neurons with graded response have collective computational properties like those of two-state neurons, Proc. Natl. Acad. Sci. USA, 81 (1984), 3088-3092 1

[10] H.-J. Jiang, J.-D. Cao, BAM-type Cohen-Grossberg neural networks with time delays, Math. Comput. Modelling, 47 (2008), 92-103.

[11] B. Kosko, Bi-directional associative memories, IEEE Trans. Systems Man Cybernet., 18 (1988), 49-60. 1 
[12] B. Kosko, Neural networks and fuzzy systems: a dynamical systems approach to machine intelligence/book and disk, Prentice Hall, Englewood Cliffs, NJ, (1992). 1

[13] Y.-K. Li, Global exponential stability of BAM neural networks with delays and impulses, Chaos Solitons Fractals, 24 (2005), 279-285.

[14] Y.-K. Li, X.-L. Fan, Existence and globally exponential stability of almost periodic solution for Cohen-Grossberg BAM neural networks with variable coefficients, Appl. Math. Model., 33 (2009), 2114-2120. 1

[15] G.-Q. Peng, L.-H. Huang, Anti-periodic solutions for shunting inhibitory cellular neural networks with continuously distributed delays, Nonlinear Anal. Real World Appl., 10 (2009), 2434-2440. 1

[16] F.-J. Qin, X.-J. Yao, Existence and exponential stability of the anti-periodic solutions for a class of impulsive Cohen-Grossberg neural networks with mixed delays, (Chinese) Comput. Eng. Softw., 5 (2014), 17-24.

[17] J.-Y. Shao, Anti-periodic solutions for shunting inhibitory cellular neural networks with time-varying delays, Phys. Lett. A, 372 (2008), 5011-5016.

[18] Q. Wang, Y.-Y. Fang, Existence of anti-periodic mild solutions to fractional differential equations of order $\alpha \in(0,1)$, Ann. Differential Equations, 3 (2013), 346-355.

[19] R. Wu, An anti-periodic LaSalle oscillation theorem, Appl. Math. Lett., 21 (2008), 928-933. 1

[20] Y.-H. Xia, Z.-K. Huang, M.-A. Han, Exponential p-stability of delayed Cohen-Grossberg-type BAM neural networks with impulses, Chaos Solitons Fractals, 38 (2008), 806-818. 1

[21] J.-Z. Xu, Z.-F. Zhou, Existence and uniqueness of anti-periodic solutions to an $\mathrm{n}$ th-order nonlinear differential equation with multiple deviating arguments, Ann. Differential Equations, 28 (2012), 105-114. 1

[22] X.-J. Yang, A new integral transform method for solving steady heat-transfer problem, Therm. Sci., 20 (2016), S639-S642. 1

[23] X.-J. Yang, A new integral transform operator for solving the heat-diffusion problem, Appl. Math. Lett., 64 (2017), 193197.

[24] X.-J. Yang, F. Gao, A new technology for solving diffusion and heat equations, Therm. Sci., 21 (2017), 133-140. 1

[25] F.-J. Yang, C.-L. Zhang, D.-Q. Wu, Global stability analysis of impulsive BAM type Cohen-Grossberg neural networks with delays, Appl. Math. Comput., 186 (2007), 932-940. 1

[26] H. Zhang, W.-T. Wang, B. Xiao, Exponential convergence for high-order recurrent neural networks with a class of general activation functions, Appl. Math. Model., 35 (2011), 123-129. 1

[27] Z.-Y. Zheng, Q. Wang, Three anti-periodic solutions of nonlinear neutral functional differential equations with variable parameter, Math. Appl. (Wuhan), 26 (2013), 198-204. 1

[28] F.-Y. Zheng, Z. Zhan, C.-Q. Ma, Periodic solutions for a delayed neural network model on a special time scale, Appl. Math. Lett., 5 (2010), 571-575. 1 